\title{
Optimization models to locate health care facilities
}

\author{
$\underline{\text { M. Dzator }}^{\mathrm{a}, \mathrm{c}}$ and J. Dzator ${ }^{\mathrm{b}, \mathrm{c}}$ \\ ${ }^{a}$ SAE, Central Queensland University, Mackay; ${ }^{b}$ The University of Newcastle, NSW \\ ${ }^{c}$ Australia Africa University Network Partner \\ Email:
}

\begin{abstract}
The rapid growth of population in cities and major regional areas, shorter length of stays in hospitals, ageing (and the desire of the elderly to stay longer in their homes), and traffic poses a challenge to health departments in meeting the demand for preventive, health center and emergency services. The changes in factors such as urbanization, demography and the rate of service utilization may affect the optimal distances or cost between patients and health care facilities. In addition, rapid population growth, increasing manmade and natural disasters seem to put increasing pressure on demands for timely health care.
\end{abstract}

There is a challenge in optimally locating health care facilities to enable the community to have good access to preventive, health center and emergency services. The location of health care facilities is an important aspect in health service delivery. It is therefore crucial for health care facilities to be located optimally to serve the community well. Facility location models have a greater importance when applied to the location of health care facilities because improper location will have a serious impact on the community.

The fundamental objectives of locating facilities can be summarized into three categories. The first category refers to those designed to cover demand within a specified time or distance. This objective gives rise to location problems which are known as the Location Set Covering Problem (LSCP) and the Maximal Covering Location Problem (MCLP). The LSCP seeks to locate the minimum number of facilities required to 'cover' all demand or population in an area. The MCLP is to locate a predetermined number of facilities to maximize the demand or population that is covered. The second category refers to those designed to minimize maximum distance. This results in a location problem known as the $p$-center problem which addresses the difficulty of minimizing the maximum distance that a demand or population is from its closet facility given that $p$ facilities are to be located. The third category refers to those designed to minimize the average weighted distance or time. This objective leads to a location problem known as the $p$-median problem. The $p$-median problem finds the location of $p$ facilities to minimize the demand weighted average or total distance between demand or population and their closest facility.

The objective of this study is to discuss the importance of the application of optimization models (maximal covering location and the $p$-median models) to locate health care facilities. We apply the $p$-median models and the maximal covering location models to real data from Mackay metropolitan area in Queensland, Australia. We compare the two models using the real data and with existing ambulance stations. The study shows that the $p$-median model gives a better solution than the maximal covering location model. We also noted that the results of the maximal covering location model depend on the pre-determined weighted coverage distance.

Keywords: Optimization, models, healthcare, facilities 


\section{INTRODUCTION}

The location of facilities is important in a good health care delivery in a community. Health care services can be classified as preventive, health center and emergency service. The delivery of most public services involves direct contact between the service facility and the target population. The location of health care facilities is one of those public facilities in which the proximity to the target population is very important. Often communities in a service area desire a closer location of facilities such as hospitals, health centers, ambulance stations, fire stations, post offices, and schools in their service area. Due to scarcity of resources, a limited number of facilities tend to be located to serve larger settlement areas and this often leads to efficiency and effectiveness problems in terms of service delivery. The application of optimization models to locate facilities is therefore crucial in service delivery. A health care facility which is difficult to access leads to increase morbidity and mortality.

In the service industries, the location of health care facilities such as ambulance stations, emergency centers, clinics and hospitals impact significantly on the safety and well-being of the community. The performance of these services can be improved by either improving the existing location using optimization models or increasing the number of facilities. However, increasing the number of facilities is generally limited or impossible due to capital constraints. It is therefore important to locate health care facilities effectively and efficiently. The main concern of location problems is to place facilities to optimize some spatially dependent objectives such as minimize average travel time or distance between demand points and servers and minimize a cost function of travel or response time.

The LSCP, MCLP and the $p$-Median problem form the basis of models in health care application (Daskin and Dean, 2004). The LSCP minimizes the number of facilities selected so that all demand points are covered (Toregas et al., 1971). The cost of covering all demand nodes is often expensive. The MCLP maximizes the number of demands covered (Church and ReVelle, 1974). The $p$-Median problem minimizes the weighted distance between the demand and the closest facility (Hakimi, 1964,1965).

A number of authors have used optimization models to locate health care facilities. These include Dzator and Dzator (2018), Beheshtifar and Alimoahmmadi (2015), Fo and Mota (2012), Shariff et al. (2012), Griffin et al. (2008), Marianov and Taborga (2001). The detail of health care facilitity location is presented in Ahmadi-Javid et al. (2017) and Afshari and Peng (2014).

The objectives of this study are to discuss the important of discrete facility location models and to compare the $p$-median model with the maximal covering location model when applied to real data. We will also investigate the effect of a pre-determined coverage distance on the $p$-median and the maximal covering location models. The rest of paper is organized as follows. We briefly discuss two primary discrete location models in Section 2. We discuss Mackay metropolitan area and present Mackay data in Section 3. Computational results obtained from Mackay data are presented in Section 4 and conclusion in Section 5.

\section{DISCRETE LOCATION MODELS}

In this section we will present the Maximal Covering Location Problem (MCLP) and the $p$-Median Problem. The facility location problem has been modeled as maximal covering location problem; and the $p$-median problem. The general problem of locating facilities is to establish them to optimize some quantities such as distance, travel time, cost and demand satisfaction. We briefly outline below the models for two discrete location models.

We define the following notation:

$I=\{1, \ldots, m\}$, the set of demand locations,

$J=\{1, \ldots ., n\}$, candidate sites for facilities,

$d_{i j}=$ the shortest distance between location $i$ and location $j$,

$x_{i j}=1$ if the customer at location $i$ is allocated to facility at location $j, 0$ otherwise,

$y_{j}=1$ if a facility is established at location $j, 0$ otherwise,

$p=$ the number of facilities to be established,

$x_{j}=1$ if a server is stationed at $j, 0$ otherwise,

$y_{i}=1$ if node $i$ is covered, 0 otherwise,

$S=$ time or distance standard for coverage,

$N_{i}=$ the set of nodes $j$ located within the standard distance or time from demand node $i$, 
$a_{i}=$ the population at demand node $i$

\subsection{Maximal Covering Location Problem (MCLP)}

Church and Revelle (1974) introduce the maximal covering location problem to address a budgetary constraint of locating a fixed number of facilities in an area. The objective of the MCLP is to locate a predetermined number of facilities, $p$, in a way as to maximize the demand that is covered.

The maximal covering location problem (MCLP) can be formulated as follows:

$$
\begin{gathered}
\text { Maximize } \sum_{i \in I} a_{i} y_{i} \\
\text { subject to: } y_{i} \leq \sum_{j \in N_{i}} x_{j}, \forall i \in I \\
\\
\sum_{j \in J} x_{j}=p \\
x_{j}, y_{i} \in\{0,1\}, \forall j \in J i \in I
\end{gathered}
$$

In the above formulation, the objective stated in equation (1) maximizes the sum of covered demands. The expression (constraint) in (2) indicates that demand $i$ can only be covered if at least one server is located within the time or distance standard $S$. Constraint (3) indicates that the total number of facilities that can be located is p. Constraint (4) are the integrality constraints on decision variables.

Megiddo et al. (1983) showed that the maximal covering location problem is NP-hard.

\subsection{The $p$-Median Problem}

Hakimi $(1964,1965)$ introduced the $p$-median problem when he considered the optimal location of switching centers in a communication network. The objective of this problem is to find the locations of $p$ facilities to minimize the demand weighted total distance (total cost) between each demand node and the nearest facility. For the $p$-median problem the cost of serving demands at node $i$ is the product of the demand at node $i$ and the distance between demand node $i$ and the nearest facility to node $i$.

We formulate the $p$-median model as follows:

$$
\begin{aligned}
& \text { Minimize } \sum_{i \in I} \sum_{j \in J} d_{i j} x_{i j} \\
& \text { subject to: } \sum_{j \in J} x_{i j}=1, \forall i \in I \\
& \sum_{j \in J} y_{j}=p \\
& x_{i j} \leq y_{j}, \forall i \in I, \forall j \in J \\
& y_{j} \in\{0,1\}, x_{i j} \in\{0,1\}
\end{aligned}
$$

The objective (5) is to minimize the total distance from customers or clients to their nearest facility. Constraint (6) shows that the demand of each customer or client must be met. From constraint (7), the number of facilities to be located is $p$. Constraint (8) shows that customers must be supplied from open facility. Constraint (9) present the problem as a binary integer programming. The above formulation assumes that the potential facility sites are nodes on the network. Hakimi (1964) however showed that allowing facilities to be located on the arcs of the network instead of the nodes would not reduce total travel cost. Garey and Johnson (1979) showed that the $p$-median problem is $N P$-hard for variable values of $p$.

\section{MACKAY METROPOLITAN AREA AND DATA}

The Mackay metropolitan comprises of 24 suburbs with a total population of 77,137 in the 2016 census. It has two ambulance stations: Mackay Ambulance Station, which is located at Paget, and North Mackay Ambulance Station, located at Beaconsfield (shown in Figure 1). The suburbs are separated into those north of the river (North Mackay, Andergrove, Beaconsfield, Blacks Beach, Bucasia, Cremorne, Dolphin Heads, Eimeo, Erakala, Glenella (with Foulden), Mackay Harbour, Mount Pleasant, Richmond, Rural View (with Nindaroo), Shoal Point and Slade Point) and those south of the river (Mackay City, East Mackay, West Mackay, South Mackay, Oooralea, Paget, Racecourse and Te Kowai). There is one major public hospital located at West Mackay. 


\subsection{Data}

For this study the distance matrix from node to node is determined by distance data using Google maps. This distance is the road network among the various suburbs in the Mackay metropolitan area. Hence the distance values are the shortest road travel distances (equivalent to distance on a road map) between the origin and the destination.

We weighted the distance by taking into consideration only the population of the origin suburb since we can also weigh by considering the nature of the road. The fact is that the better the road network the easier it is for a vehicle to move from one suburb to another. The weighted distance is thus the product of the population at the origin suburb and the distance between the origin suburb and the destination suburb. We note that the larger the population the larger the weight. This weighted distance is calculated for each of the twenty-four suburbs in the Mackay Metropolitan region. This calculation is done by noting the distance from a suburb to all other suburbs and each value is multiplied by the weight of the origin suburb. This is repeated for every suburb and values are recorded as a column matrix representing Mackay Metropolitan area. The population for the suburbs which was used for the weight for each node ranges from 33 for a suburb of Cremorne to 9419 which corresponds to Andergrove and this shown in Table 1. The minimum weighted distance for the Mackay Metropolitan area is $66 \mathrm{~km}$ and the maximum value is $178,068 \mathrm{~km}$.

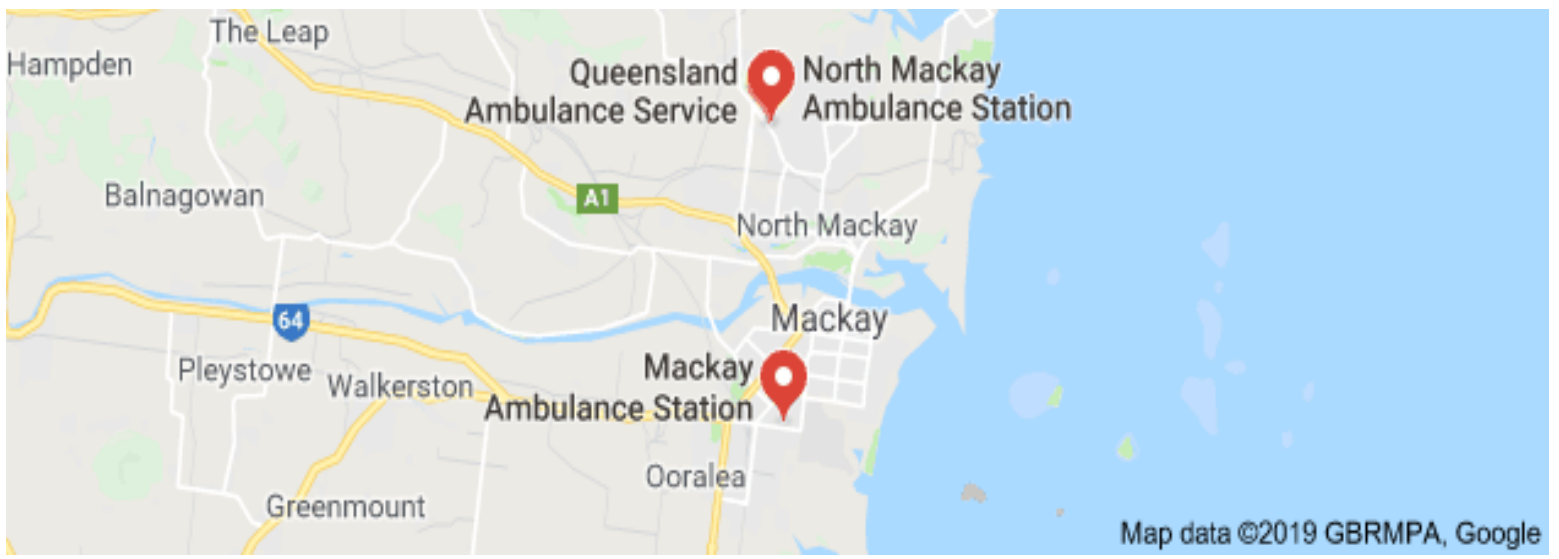

Figure 1. Ambulance Stations in Mackay. Source:

https://www.google.com/search?rlz=1C1GGRV_enAU751AU751\&q=Ambulance+stations+in+Mackay

Table 1. Names of suburbs in Mackay Metropolitan Area and their Population from 2016 Census Data

\begin{tabular}{|l|l|l|l|l|l|}
\hline Node & Suburb & Population & Node & Suburb & Population \\
\hline 1 & Mackay City & 3659 & 13 & Erakala & 547 \\
\hline 2 & East Mackay & 3564 & 14 & Glenella (with Foulden) & 4519 \\
\hline 3 & West Mackay & 6210 & 15 & Mackay Harbour & 555 \\
\hline 4 & North Mackay & 5933 & 16 & Mount Pleasant & 4444 \\
\hline 5 & South Mackay & 6706 & 17 & Ooralea & 3366 \\
\hline 6 & Andergrove & 9419 & $18 *$ & Paget & 273 \\
\hline $7^{*}$ & Beaconsfield & 5490 & 19 & Racecourse & 184 \\
\hline 8 & Blacks Beach & 3861 & 20 & Richmond & 648 \\
\hline 9 & Bucasia & 4771 & 21 & Rural View (with Nindaroo) & 4793 \\
\hline 10 & Cremorne & 33 & 22 & Shoal Point & 977 \\
\hline 11 & Dolphin Heads & 388 & 23 & Slade Point & 3349 \\
\hline 12 & Eimeo & 3230 & 24 & Te Kowai & 218 \\
\hline
\end{tabular}

\section{COMPUTATIONAL RESULTS}

We used the SITATION software (Daskin; 1995) to determine the results for the $p$-median and the MCLP locations for different weighted coverage distance. The first two existing locations are known so we determined their objective values using the SITATION software. Facilities are then added optimally to existing locations using the SITATION software and we noted the objective value for the locations. We used the Lagrangian relaxation to determine the results for the $p$-median problem and MCLP. The detail of the method is discussed in Daskin (1995). 
Table 2. The $p$-Median Location and Maximal Covering Location for $70 \mathrm{~km}$ to $500 \mathrm{~km}$ Weighted Coverage Distance

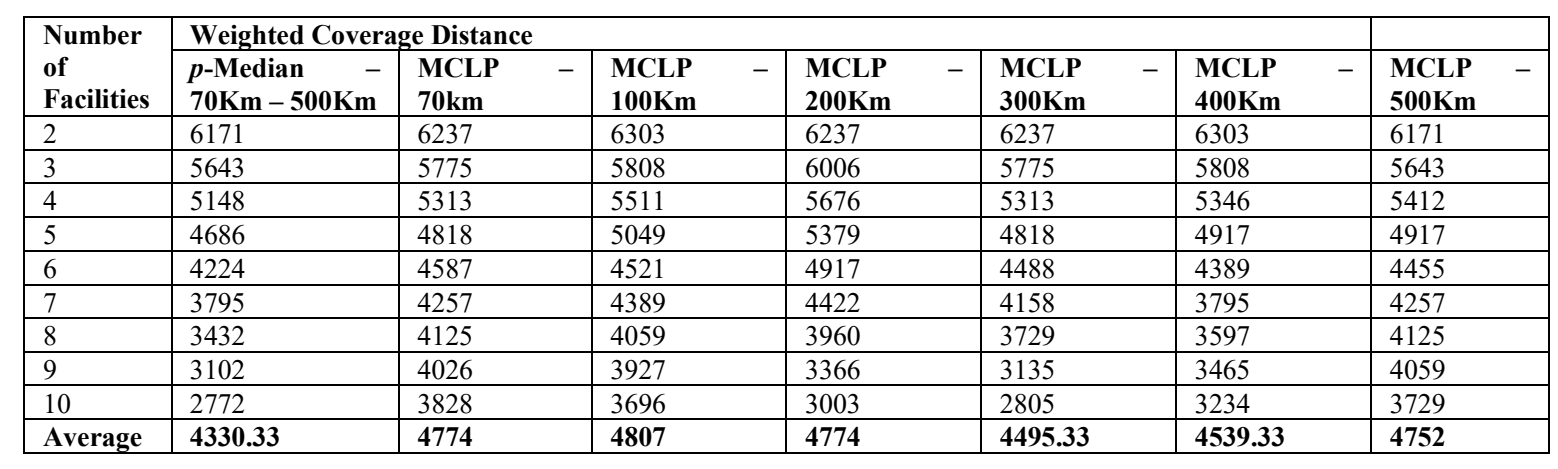

Table 3. Comparison of Existing Location with the $p$-Median Problem and MCLP

\begin{tabular}{|c|c|c|c|c|c|c|}
\hline Number of Facilities & EL Cost & $\begin{array}{l}p \text {-Median } \\
\text { Cost }\end{array}$ & $\begin{array}{l}\text { MCLP Cost } \\
(300 \mathrm{Km})\end{array}$ & $\begin{array}{l}\text { Cost } \quad \text { Change } \\
\text { Using } p \text {-Median }\end{array}$ & $\begin{array}{l}\text { Cost Change Using } \\
\text { MCLP }\end{array}$ & $\begin{array}{lr}\text { Cost } & \text { Saving } \\
\text { Using } & p- \\
\text { Median } & \\
\end{array}$ \\
\hline 2 & 82173 & 6171 & 6237 & 76002 & 75936 & 66 \\
\hline 3 & 6237 & 5643 & 5775 & 594 & 462 & 132 \\
\hline 4 & 5643 & 5148 & 5313 & 495 & 330 & 165 \\
\hline 5 & 5115 & 4686 & 4818 & 429 & 297 & 132 \\
\hline 6 & 4620 & 4224 & 4488 & 396 & 132 & 264 \\
\hline 7 & 4158 & 3795 & 4158 & 363 & 0 & 363 \\
\hline 8 & 3696 & 3432 & 3729 & 264 & -33 & 297 \\
\hline 9 & 3267 & 3102 & 3135 & 165 & 132 & 33 \\
\hline 10 & 2904 & 2772 & 2805 & 132 & 99 & 33 \\
\hline
\end{tabular}

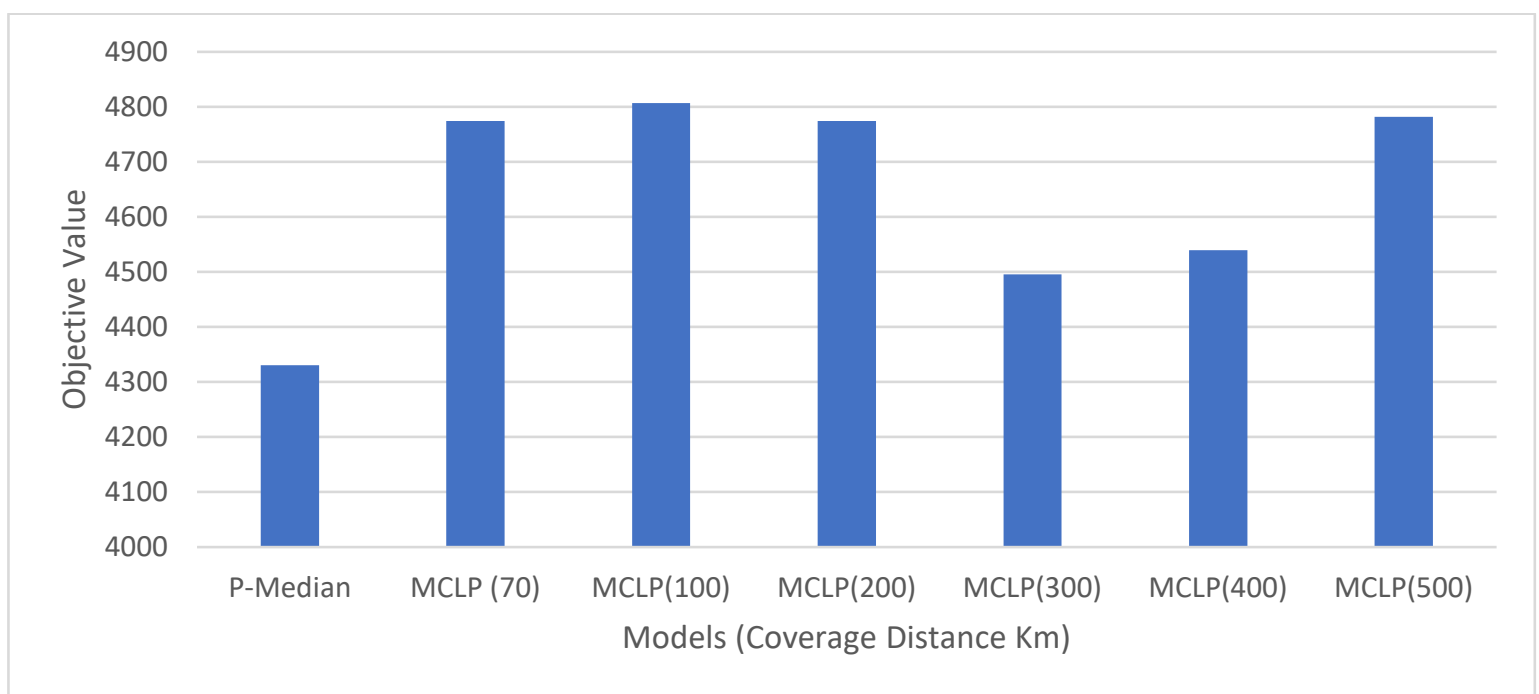

Figure 2. The Objective Value of the $p$-Median Problem and MCLP with different Coverage Distance

Table 2 shows the results of the $p$-median problem and the MCLP, The average of the objective values for two to ten facilities are as follows: $p$-median problem is 4330.33, MCLP with weighted coverage distance of $300 \mathrm{~km}$ is 4495.33; MCLP with weighted coverage distance of $400 \mathrm{~km} 4539.33$, MCLP with weighted coverage distance of $500 \mathrm{~km}$ is 4752 , MCLP with weighted coverage distance of $70 \mathrm{~km}$ is 4774 , MCLP with weighted coverage distance of $100 \mathrm{~km}$ is 4807 .

Figure 2 clearly shows that the $p$-median problem gives the best result followed by the MCLP with weighted coverage distance of $300 \mathrm{~km}$, MCLP with weighted coverage distance of $400 \mathrm{~km}$ and the worst result is given by MCLP with weighted coverage distance of $100 \mathrm{~km}$. We noted that as we change the coverage distance the results of the MCLP changes while that of the $p$-median problem remains the same for the facilities that are located. 
In Table 3 the objective value of the existing locations is compared with the objective value of $p$-median problem and the MCLP. While the $p$-median problem peforms better in all cases when facilities are located the existing locations performs better than MCLP when eight facilities are located and is the same when seven facilitires are located. The cost saved if the $p$-median problem is used instead of MCLP when two to ten facilities are located ranges from 33 to 363 .

\section{CONCLUSION}

In this paper we discussed two discrete location models namely the MCLP and the $p$-median problem and we outline the important of locating health care facilities using optimization models. We applied the models to real data obtained by calculating the weighted distance in Mackay metropolitan using 2016 population data. The results we obtained show that the $p$-median problem performs better than the MCLP. However, both models perform better than the existing locations. It therefore necessary to use optimization model to locate healthcare facilities for proper accessibility and cost savings.

\section{REFERENCES}

Afshari, H. and Peng, Q. (2014). Challenges and Solutions for Location of Healthcare Facilities, Industrial Engineering and Management, 127 (3), doi: 10.4172/2169-0316.1000127

Ahmadi-Javid, A., Seyedi, P. and Syam, S.S. (2017). A survey of healthcare facility location, Computers \& Operations Research, 79, 223-263.

Beheshtifar, S. and Alimoahmmadi, A. (2015). A multiobjective optimization approach for location-allocation of clinics, International Transactions in Operational Research, 22, 313-328

Church, R. and ReVelle, C. (1974), The maximal covering location problem, Papers of the Regional Science Association, 32, 101-118.

Daskin, M.S. (1995), Network and Discrete Location: Models, Algorithms and Applications, John Wiley and Sons, Inc., 498 pp., New York.

Daskin, M.S. and Dean, L.K. (2004). Location of health care facilities. In Brandeau, M.L., Sainfort, F., Pierskalla, W.P. (eds), Operations Research and Health Care. Kluwer Academic Press, Boston, MA, 4376.

Dzator, M. and Dzator, J. (2018). The $p$-median problem and health facilities: cost saving and improvement in healthcare through facility location, In Sarker, R., Abass, H.A., Dunstal, S., Kilby, P., Davis, R., Young, L. (eds), Data and Decision Sciences in Action. Springer, 369-380.

Fo, A. and Mota I. (2012). Optimization models in the location of healthcare of healthcare facilities: a real case in Brazil, Journal of Applied Operational Research, 4(1), 37-50.

Garey, M.R and D.S. Johnson (1979), Computers and Intractability: A Guide to the Theory of NPCompleteness, Prentice-Hall, Englewood Cliffs, NJ.

Griffin, P.M., Scherrer, C.R. and Swann, J.L. (2008). Optimization of community health center locations and service offerings with statistical need estimation, IIE Transactions, 40(9), 880-892.

Hakimi, S.L. (1964), Optimisation locations of switching centres and the absolute centres and medians of a graph, Operations Research, 12, 450-459.

Hakimi, S.L. (1965), Optimum distribution of switching centers in a communication network and some related graph theoretic problems, Operations Research, 13, 462-475.

Marianov, V. and Taborga, P. (2001). Optimal location of public health centres which provide free and paid services, Journal of the Operational Research Society, 52, 391-400.

Megiddo, T.H., E. Zemel and S.L. Hakimi (1983), The maximum coverage location problem, SIAM Journal of Algebraic and Discrete Methods, 4, 253-261.

Shariff, S., Moin, M.H. and Omar, M. (2012). Location allocation modeling for healthcare facility planning in Malaysia, Computers \&Industrial Engineering, 62, 1000-1010.

Toregas, C., R. Swain, C. ReVelle, and L. Bergman (1971), The location of emergency service facilities, Operations Research, 19, 1363-1373. 